\title{
Colloidal crystals model real world
}

Neat Australian experiments have shown that an artificial three-dimensional colloid can be a good model of the real world of crystalization in the lab as well as on paper.

EVERYBody would like to know more about two of the most common phenomena in physical science - the melting of solids and the inverse process, their crystallization. Of course, the gross features of each process are easily described. A pure material melts at a particular temperature, called the melting point. There is a positive latent heat and, given information about the compressibility of the liquid and solid phases, it is possible to calculate the change of the melting point with pressure. Simple thermodynamics, that is, first done 150 years ago by William Thomson (later Lord Kelvin).

The trouble is that this stark statement cannot apply to what happens on a microscopic scale. Improperly to put the difficulty in anthropomorphic terms, how does a whole crystal being warmed know that the point is approaching when each of its atoms or molecules is to forsake imprisonment on the crystal lattice for the freedom to move about at will? Luckily, there is a means of communication within all crystals: the lattice vibrations which, quantized by the procedures worked out by Max Born and others in the 1930s, each involve every atom in the crystal. But if that is how the warning is delivered, how can inanimate atoms and molecules remember what it is?

Part of the answer no doubt lies in phrases such as the 'roughening transition', the observation that physical changes in the surface of a solid crystal appear to show up in advance of melting in the strict sense. It looks as if some parts of the crystal are preparing (more anthropomorphism) to break away. But dutifully they wait until the whole crystal is ready, and only then they go free. It would be an interesting exercise to calculate what blend of photons sets this process in train.

On the face of things, crystallization should be more easily understood, but that is not what closer inspection shows. Crystals cannot appear as entire objects, but must grow, for one thing. That requires a register for the regularity of their lattices, which must be a nucleation centre. That is a microcrystal of some kind, perhaps just a cluster of a few atoms or molecules formed stochastically in the melt, perhaps a crystal of some more refractory material that happens to have similar lattice constants or perhaps just a roughness of appropriate microscopic dimensions on the boundary walls of the container. Another difficulty (but really it is support for the doctrine of nucleation) is that supercooling of a liquid below the melting point is commonplace.

Over the years, the concept of nucleation has been recognized as a prerequisite of crystallization. The trouble here is that it has hardly ever been observed. Especially if there is supercooling, a crystal will form from a melt too quickly to be followed with all the high-speed cameras in the world (but people keep trying). And from time to time, scraps of new information do nevertheless come to light.

A band of brothers at the Royal Melbourne College of Technology has meanwhile, over at least a decade, been cultivating a model of the process of crystallization that stands in relation to the real thing as does, say, the behaviour of fluid vortices in a rotating drum to the evolution of the weather in the atmosphere of the spinning Earth. Their starting point is the notion that the slow formation of crystalline aggregates in a suspension of colloidal particles is a representation of what happens when melts are cooled. The difference is that the speed with which ordered structures form in colloidal suspensions is slow enough to be followed at leisure.

First, then, make your colloid. W. van Megen, apparently the leader of this enterprise, settled some years ago for colloids made from polymethylmethacrylate spheres (400 $\mathrm{nm}$ in diameter, or no bigger than a wavelength of red light), coating them with a $10 \mathrm{~nm}$ layer of covalently bound macromolecules to ensure repulsive forces between neighbouring spheres and good interaction with the solvent.

In earlier reports (P. N. Pusey \& W. van Megen Nature 320, 340-342; 1986 and W. van Megen \& S. M. Underwood Nature 362, 616-618; 1993), the group had effectively established that the analogy between the formation of ordered structures of these spheres and the process of crystallization in real molten solids is more or less exact. Now they have gone a step further, and have shown not merely that nucleation is an essential feature of crystallization, but that nuclei form more quickly as crystallization proceeds (J. L. Harland, S. J. Henderson, S.M. Underwood \& W. van Megen Phys. Rev. Lett. 75, 3572-3575; 6 November 1995).

What happens with a colloid of this kind depends crucially on the volume fraction of the colloid particles, in this case the polymethylmethacrylate spheres. If the fraction exceeds 0.58 , the colloid forms a glass: the spherical particles cannot diffuse around each other. In a more dilute suspension with a volume fraction less (but not too much less) than 0.494 , on the other hand, the particles form a regular array as if they were part of a crystal; that is the freezing point. The melting point (but remember that the variable is not temperature but volume fraction or concentration) lies above the freezing point and below the glass transition.

The interest of what follows is that it is possible actually to carry out measurements. Indeed, the trick is to use the scattering of laser light from a monochromatic laser as if it were an X-ray beam used in Bragg diffraction mode. The system has then to be calibrated by recording the signal from incoherently scattered light with an array of photodetectors. A cubical glass box one centimetre in all directions makes the working laboratory space from which light is scattered and otherwise collected. Because the colloidal particles hold themselves in crystalline arrays only tenuously, it is child's play to reduce crystal to its constituent polymethylmethacrylate spheres; simply arrange to have the $1-\mathrm{cm}$ cube box turned end over end for a day or so, whereupon whatever it may contain will fall apart under shear stress.

Unpromising though the analogy between an ordered colloidal structure and a crystal may be, the outcome of the measurements is encouraging. Much as in the real world of molecular crystals, colloidal crystals form at such a pace that the nucleation rate appears to increase as the volume not occupied by ordered colloidal particles shrinks. That appears to accord with what has been calculated about the properties of plastic spheres in a solvent with which they interact.

What is to be learned from studies of this kind? A little and a lot. The simplest truth is that nucleation does indeed play a crucial role in crystallization. It is also unsurprising that, with the passage of time, the volume density of ordered patches of crystallinity at first grows them shrinks; crystals are created at the nuclei formed in increasing numbers, but then also begin to swallow up each other. But what is hard to understand is that the rate at which crystallization nuclei appear seems to increase as the material from which they may be made shrinks in volume. As yet, there is only hand-waving to sustain that point.

John Maddox 